\title{
Bufalin reverses acquired drug resistance by inhibiting stemness in colorectal cancer cells
}

\author{
JIAN SUN ${ }^{1 *}, \mathrm{KE} \mathrm{XU}^{1 *}$, YANYAN QIU $^{1}, \mathrm{HONG} \mathrm{GAO}^{1}$, JIANHUA XU $^{1,3}$, \\ QINGFENG TANG ${ }^{1,2}$ and PEIHAO YIN ${ }^{1,4}$ \\ ${ }^{1}$ Interventional Cancer Institute of Integrative Medicine and Putuo Hospital, \\ ${ }^{2}$ Experimental Research Center, Departments of ${ }^{3}$ Clinical Oncology and ${ }^{4}$ General Surgery, \\ Putuo Hospital, Shanghai University of Traditional Chinese Medicine, Shanghai 200062, P.R. China
}

Received January 24, 2017; Accepted June 12, 2017

DOI: $10.3892 /$ or.2017.5826

\begin{abstract}
Drug resistance is an obstacle to chemotherapy in tumor patients. Recent studies have shown that the high stemness of cancer cells may be induced by chemotherapeutic drugs, which is correlated with drug resistance. In the present study, we investigated the effects of bufalin on the stemness of colorectal cancer. We found that cisplatin could induce high stemness through the tumorsphere formation assay in vitro and in vivo in the colorectal cancer cell lines HCT116 and LoVo. In addition, cisplatin-treated tumorsphere cells showed drug-resistant properties. These results suggested that acquired drug resistance induced by cisplatin in colorectal cancer cells occurred via high stemness. On assessing the effects of bufalin, a traditional Chinese medicine monomer, we found that it could reverse the high stemness and drug resistance induced by cisplatin in colorectal cancer. These findings suggest that bufalin plays an adjuvant role in colorectal cancer chemotherapy and may help reverse acquired drug resistance. These findings may aid in the development of new therapeutic strategies.
\end{abstract}

\section{Introduction}

Although long-term declines in colorectal cancer (CRC) incidence and mortality rates have been reported in the United States $(1,2)$, these rates have increased rapidly in several areas including Spain, Eastern Europe, and China (3-5). Presently, $\mathrm{CRC}$ is the third most common malignant tumor in the world (6). Although chemotherapeutic drugs are used exten-

Correspondence to: Professor Peihao Yin or Dr Qingfeng Tang, Interventional Cancer Institute of Integrative Medicine and Putuo Hospital, Shanghai University of Traditional Chinese Medicine, 164 Lanxi Road, Putuo, Shanghai 200062, P.R. China

E-mail: yinpeihao1975@hotmail.com

E-mail: tangqingfeng126@126.com

${ }^{*}$ Contributed equally

Key words: bufalin, drug resistance, colorectal cancer, cisplatin, stemness sively for treating CRC, drug resistance is a major obstacle to the success of cancer chemotherapy. Therefore, it is important to identify a new drug capable of overcoming chemotherapy resistance in CRC patients (7).

Recent studies have highlighted that cancer stem cells (CSCs) are responsible for chemotherapy resistance $(8,9)$. According to the currently accepted and putative definition, CSCs have the capacity of self-renewal and differentiation, stress and drug resistance, and enhanced migration $(10,11)$. Colorectal CSCs have been identified and isolated from CRCs $(12,13)$. The stemness of colorectal CSCs was identified to be associated with specific properties such as high expression of CD133, CD34, ALDH, NANOG, OCT4, and SOX2 (13-16). The overexpression of these molecules is often related to the drug resistance of tumors (17). Considering the chemotherapy resistance role of the stemness of CSCs, stemness markers have become new therapeutic targets in CRC patients $(7,18)$.

Bufalin, a traditional Chinese medicine monomer, is a major active ingredient isolated from the traditional Chinese medicine Chansu (19). In the past decade, bufalin was shown to possess high anticancer ability in various cancers (20-24). The anticancer mechanisms of bufalin can be summarized as: inhibition of proliferation (20), promotion of apoptosis (24), inhibition of angiogenesis and metastasis (21), reversal of drug resistance (23), and induction of autophagy (25). Recent studies have suggested that bufalin inhibits differentiation, proliferation, and drug resistance in cancers via the inhibition of stemness (26-28). According to studies on bufalin and colorectal CSCs, signal pathways regulated by bufalin such as Wnt/ß-catenin (29), PI3K/AKT (30), Jak/STAT3 (31), Hedgehog (28), and Notch (27) are correlated with the stemness of CRC (32). Therefore, we speculated that bufalin reverses drug resistance via the inhibition of the stemness of CRC.

In the present study, we investigated the effects of bufalin on the stemness of CRC. We hypothesized that bufalin inhibits the stemness induced by cisplatin and increases the therapeutic effect of cisplatin in CRC.

\section{Materials and methods}

Cell culture. Human CRC cell lines, including HCT116 and LoVo, were cultured in RPMI-1640 medium (Gibco 
Laboratories, Grand Island, NY, USA) supplemented with $10 \%$ fetal calf serum (Gibco Laboratories) at $37^{\circ} \mathrm{C}$ in a $5 \% \mathrm{CO}_{2}$ humidified atmosphere.

Reagents and antibodies. Cisplatin was purchased from Qilu Pharmaceutical (Jinan, China). Bufalin was purchased from Sigma (St. Louis, MO, USA). CD44 (60224-1-IG), CD133 (18470-1-IG), OCT4 (11263-1-AP), SOX2 (11064-1-AP), and NANOG (14295-1-AP) primary antibodies were purchased from Proteintech (Chicago, IL, USA). GAPDH (\#2118) and ABCG2 (\#42078) were purchased from Cell Signaling Technology, Inc. (Danvers, MA, USA).

Cell viability assays. Cells were seeded in a 96-well plate at a density of $1 \times 10^{4}$ cells/well. Cell viability assays used the Cell Counting Kit-8 (CCK-8, Dojindo Laboratories, Kumamoto, Japan). Cell viability was evaluated by determining the absorbance of each well at $450 \mathrm{~nm}$ using a plate reader (Bio-Rad, Hercules, CA, USA). Each sample was analyzed in sextuplicate, and experiments were repeated thrice.

Flow cytometry. The Annexin V-FITC/PI Apoptosis Detection kit (Becton-Dickinson, Franklin Lakes, NJ, USA) was used to investigate apoptosis. Tumorsphere cells were dissociated into single cells and were then stained with Annexin and PI separately. The apoptosis ratio was assessed by flow cytometry using the FACSCalibur system (Becton-Dickinson).

The DNA-binding dye Hoechst 33342 was used to evaluate the SP ratio. Dissociated sphere cells were stained with Hoechst 33342 for $10 \mathrm{~min}$ and were tested through dual-wavelength analysis using flow cytometry (Hoechst red 675/20; Hoechst blue 424/44). SP cells were shown to have a characteristic tail, which differentiated them from other cells of the population.

The protein expression of stemness markers, such as CD133 and CD44, was detected using flow cytometry. Dissociated sphere cells were incubated with primary antibodies, including CD44 and CD133 antibodies, at $4^{\circ} \mathrm{C}$ for $1 \mathrm{~h}$. They were then washed with phosphate-buffered saline (PBS) twice and incubated with Alexa Fluor 488 conjugated anti-rabbit secondary antibodies (R37116) and Alexa Fluor 488 conjugated anti-mouse secondary antibodies (A-21202) (Invitrogen, Carlsbad, CA, USA) at $4^{\circ} \mathrm{C}$ for $30 \mathrm{~min}$ in the dark. The fluorescence values of $\mathrm{CD} 133$ and CD44 were determined using flow cytometry and analyzed using the FlowJo 7.6 software (Treestar, Inc., Ashland, OR, USA).

Immunofluorescence staining. Dissociated sphere cells were seeded on cover slips pre-coated with $0.01 \%$ polylysine at a density of 1,000 cells per well in a 48-well chamber. After $24 \mathrm{~h}$, the cells were treated with cisplatin and bufalin for $48 \mathrm{~h}$. The cells were then treated in turn with $4 \%$ paraformaldehyde for $20 \mathrm{~min}$, $0.1 \%$ Triton $\mathrm{X}-100$ for $10 \mathrm{~min}, 5 \%$ bovine serum albumin (BSA) for $60 \mathrm{~min}$, and primary antibodies overnight at $4^{\circ} \mathrm{C}$. Next, the cells were washed thrice using PBS and incubated with Alexa Fluor 488 conjugated anti-mouse secondary antibodies, Alexa Fluor 488 conjugated anti-rabbit secondary antibodies, and Alexa Fluor 555 conjugated anti-rabbit secondary antibodies (A-31572) (Invitrogen) for $1 \mathrm{~h}$. The cells were then observed using a fluorescence microscope (Leica, Wetzlar, Germany).
Western blotting. Secondary tumorspheres treated with cisplatin and bufalin were collected through centrifugation and concentration. Subsequently, tumorspheres were lysed with M-PER Mammalian Protein Extraction reagent with protease inhibitor cocktail (100X) (Sangon Biotech, China) and $1 \mathrm{mM}$ PMSF. The lysate was centrifuged at $4^{\circ} \mathrm{C}$ at $12,000 \mathrm{~g}$ for $15 \mathrm{~min}$, and the supernatant was used for western blotting. The protein concentration was measured using the Bradford Coomassie Blue G-250 method. Protein (40 $\mu \mathrm{g})$ was mixed with 5X SDS sample buffer and was denatured by boiling for $10 \mathrm{~min}$. The denatured protein was loaded onto $10 \%$ polyacrylamide SDS gels (PAGE-SDS) and transferred onto PVDF membranes (Millipore, Billerica, MA, USA). Membranes were blocked in $5 \% \mathrm{BSA}$ for $2 \mathrm{~h}$ followed by incubation with primary antibodies overnight at $4^{\circ} \mathrm{C}$. After washing thrice for $10 \mathrm{~min}$ in TBST, membranes were incubated with HRP-conjugated secondary antibodies for $2 \mathrm{~h}$ at room temperature (RT). Subsequently, the membranes were washed thrice for $10 \mathrm{~min}$ in TBST and were visualized using the ECL Western Blotting Detection system (Millipore). The ratio of the optical densities of the bands was measured using a gel image analysis system (Bio-Rad) and normalized to GAPDH.

Tumorsphere formation assays. HCT116 and LoVo cells were separately seeded in ultra-low attachment 24-well plates (Corning, Corning, NY, USA) with DMEM/F-12 (12660012, Gibco) culture media, B27 (17504044, Gibco), 20 ng/ml EGF (PHG0311, Gibco), and $20 \mathrm{ng} / \mathrm{ml}$ bFGF (13256029, Gibco) at a density of 200 cells/well. The medium was replaced by half every 3 days. After 14 days, tumorspheres were counted and photographs were obtained through microscopy.

Colony formation assays. Single cells were prepared and seeded into 96 -well plates at a density of 200 cells/well. The medium was replaced every 2 days. After 10 days, cells were treated in turn with $4 \%$ paraformaldehyde for $20 \mathrm{~min}$ and crystal for $20 \mathrm{~min}$, and were washed with PBS at least twice. The colonies were counted, and photographs were obtained through microscopy.

In vivo tumor xenograft model. For the in vivo xenograft tumor growth assay, male nude mice [BALB/c nu/nu, 5-week-old, purchased from SLAC (Shanghai Laboratory Animal Center, Shanghai, China)] were used to prepare the in vivo tumor xenograft model. Two million cells in $0.1 \mathrm{ml}$ of PBS were injected into the subcutaneous tissues of each mouse. After 2 weeks, mice were injected intraperitoneally with cisplatin $(10 \mathrm{mg} / \mathrm{kg}$ body weight) and bufalin ( $1 \mathrm{mg} / \mathrm{kg}$ body weight) every 3 days for 4 weeks. Finally, the tumor-bearing mice were sacrificed and the tumors were excised and weighed.

Immunochemistry. All tumor xenograft bodies were formalinfixed, embedded in paraffin, serially sectioned (5- $\mu \mathrm{m}$ thickness), and mounted on glass slides. The reagents in the subsequent process were purchased from Maixin Bio (Fuzhou, China). Sections were incubated for $10 \mathrm{~min}$ in peroxidase blocking agent, washed for 3 min thrice with PBS, blocked with rabbit serum for $60 \mathrm{~min}$ at RT, and incubated with antibodies at $4^{\circ} \mathrm{C}$ overnight. Subsequently, the sections were washed thrice with PBS, incubated with HRP-conjugated secondary antibodies for 
A Primary tumor sphere formation assay

Day $0 \quad$ Day 14

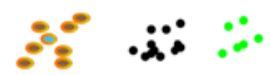

HCT116, LoVo Cis Buf

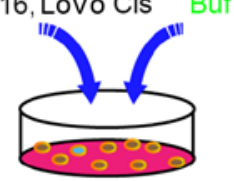

B

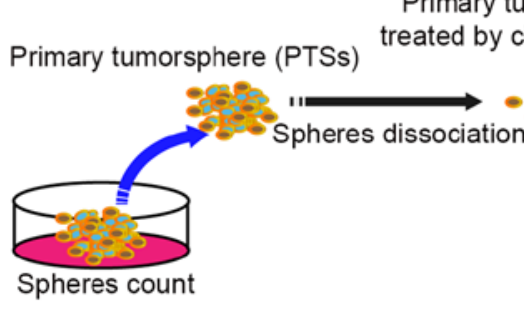

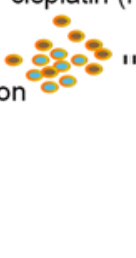

Secondary tumor sphere formation assay

- Normal cancer cells

- cscs

Cell viability and apoptosis assay for drug-resistance

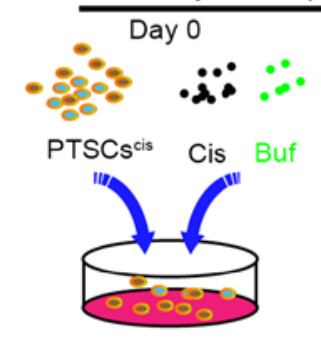

Day 14

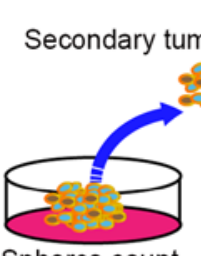

Spheres count
Secondary tumorsphere cells Secondary tumorsp
(STSs) (STSCs) $\rightarrow 2$

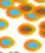

$\mathrm{SP}$ ratio assay for stemness

Test the expression of stemness marker

- Normal cancer cells

- cSCs

Figure 1. Schematic of the tumorsphere formation assay. (A) Primary tumorsphere formation assay for the effects of Cis and Buf on self-renewal of colorectal cancer cells. (B) Secondary tumorsphere formation assay for stemness and drug resistance. Cis, cisplatin; Buf, bufalin.

10 min at RT, washed again with PBS, developed with diaminobenzidine solution, and counterstained with hematoxylin. Additionally, serial sections were stained with hematoxylin and eosin.

Statistical analysis. Data are presented as mean \pm SD. All analyses were performed using the SPSS 17.0 software (IBM Corp., Armonk, NY, USA). A p-value of $<0.05$ was considered statistically significant.

\section{Results}

Previous studies suggested that the self-renewal properties of CSCs could be judged by the formation of 3D spheroids in a non-adhesive environment, which was called tumorsphere formation assay $(33,34)$. In this study, tumorsphere formation assays were used to analyze the effects of cisplatin and bufalin on stemness in two CRC cell lines (HCT116 and LoVo). Initially, HCT116 and LoVo cells were treated separately with cisplatin and bufalin at different concentrations in a non-adhesive culture system for 14 days. Subsequently, the numbers and diameters of the spheres were counted to analyze the effects of cisplatin and bufalin on the stemness of CRC cells (Fig. 1A). Then, primary tumorspheres (PTSs) treated with cisplatin $(5 \mu \mathrm{M})$ were dissociated into single cells $\left(\mathrm{PTSCs}^{\mathrm{cis}}\right.$ ) for secondary tumorsphere formation assay. The cells were treated separately with cisplatin $(5 \mu \mathrm{M})$, bufalin $(5 \mathrm{nM})$, and their combination for 14 days, and the numbers and diameters of the tumorspheres were counted. Subsequently, secondary tumorspheres (STSs) were dissociated into single cells (STSCs) for: i) cell viability and apoptosis assay for drug resistance; ii) side population (SP) ratio assay for stemness; and iii) assay for the expression of stemness markers (Fig. 1B). Recent studies found that a small population of cells differed from the main population of cancer cells on observing staining with a DNA-binding dye using flow cytometry. The small population of cells was called the SP, which was thought to be part of CSCs with CSC-like phenotypic properties.

Cisplatin enhances the tumorsphere formation capacity of colorectal cancer cells in vitro. Tumorsphere formation assay using a non-adhesive culture system is an important method for the identification of stemness in vitro $(34,35)$. To evaluate the effect of cisplatin on the stemness of CRC cells, we tested the ability of tumorsphere formation of two CRC cell lines (HCT116 and LoVo). At the same time, colony formation assay was used to evaluate the effects of cisplatin on the proliferation of these two cell lines. In order to analyze the results of the two experiments, we used the same cisplatin concentrations and the same experiment duration (14 days).

As shown in Fig. 2A-C, with increasing cisplatin concentration $(0-5 \mu \mathrm{M})$, the numbers and diameters of HCT116-PTSs ${ }^{\text {cis }}$ and LoVo-PTSs ${ }^{\text {cis }}$ increased. Therefore, cisplatin could increase tumorsphere formation of CRC cells in a dose-dependent manner within a certain concentration range. However, the numbers and diameters started to decrease when the cisplatin concentration reached $10 \mu \mathrm{M}$, and tumorspheres were not found when the cisplatin concentration reached $50 \mu \mathrm{M}$, which suggested that the anti-proliferation effects of higher concentrations of cisplatin $(10-50 \mu \mathrm{M})$ were greater than the stemness effects.

Colony formation assay using the adhesive culture system was used to analyze the anti-proliferation effects of cisplatin in this study. We found that the efficiency of colony formation 


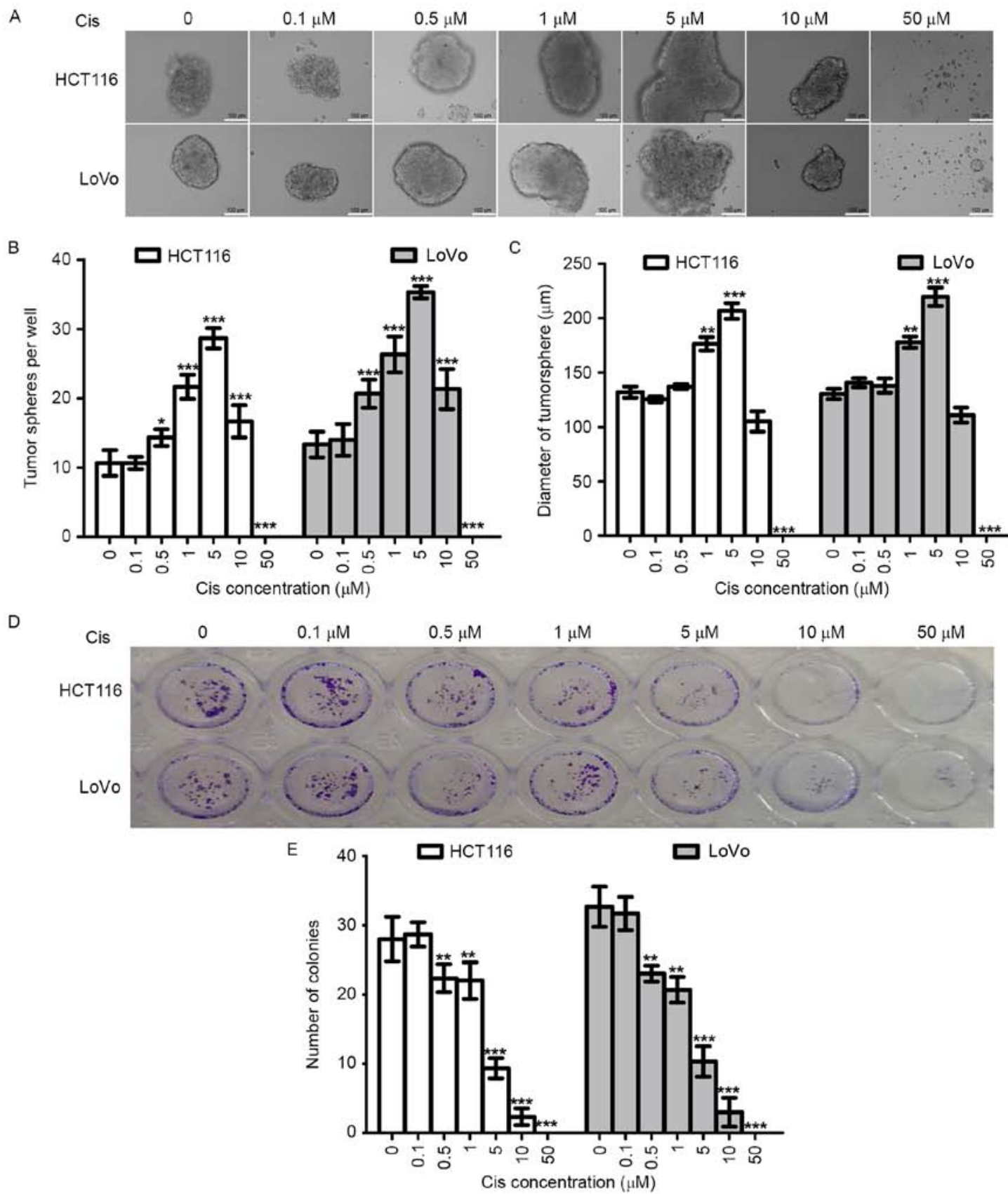

Figure 2. Effects of cisplatin on tumorsphere formation in vitro. The tumorsphere formation assay was used to analyze the effects of cisplatin $(0,0.5,1,5$, 10 and $50 \mu \mathrm{M}$ ) on the stemness of HCT116 and LoVo cells. (A) Representative image of tumorspheres obtained through microscopy. (B) Histogram of the number of tumorspheres. (C) Histogram of the mean tumorsphere diameter per well. (D) Representative image of the colony formation assay obtained through microscopy. (E) Histogram of the number of colonies. ${ }^{* * *} \mathrm{p}<0.001,{ }^{* *} \mathrm{p}<0.01,{ }^{*} \mathrm{p}<0.05$.

decreased with cisplatin treatment in a dose-dependent manner (Fig. 2D and E). When the cisplatin concentrations were $5 \mu \mathrm{M}$ and $10 \mu \mathrm{M}$, the number of colonies decreased. The results of the colony formation assay and tumorsphere formation assay were opposite with cisplatin treatment at the same concentrations and experiment durations, which further supported the increasing stemness effects of cisplatin on CRC cells.

Bufalin decreased the tumorsphere formation capacity of colorectal cancer cells in vitro. To determine the effects of bufalin on the stemness of CRC cells, we first tested the tumorsphere formation capacity of CRC cells treated with different concentrations of bufalin. We also analyzed the effects of bufalin on killing and proliferation inhibition using the colony formation assay. We found that bufalin could inhibit tumorsphere formation of HCT116 and LoVo cells in a dose-dependent manner (Fig. 3A-C). The trend of the colony formation assay results was similar to that of the tumorsphere formation assay results (Fig. 3D and E); however, $1 \mathrm{nM}$ of bufalin inhibited tumorsphere formation but not colony formation, which suggested that the inhibition of tumorsphere formation effects of bufalin relied not only on anti-proliferation but also on anti-stemness.

Bufalin is effective against cisplatin with regard to the stemness of colorectal cancer cells. In view of the anti-stemness 


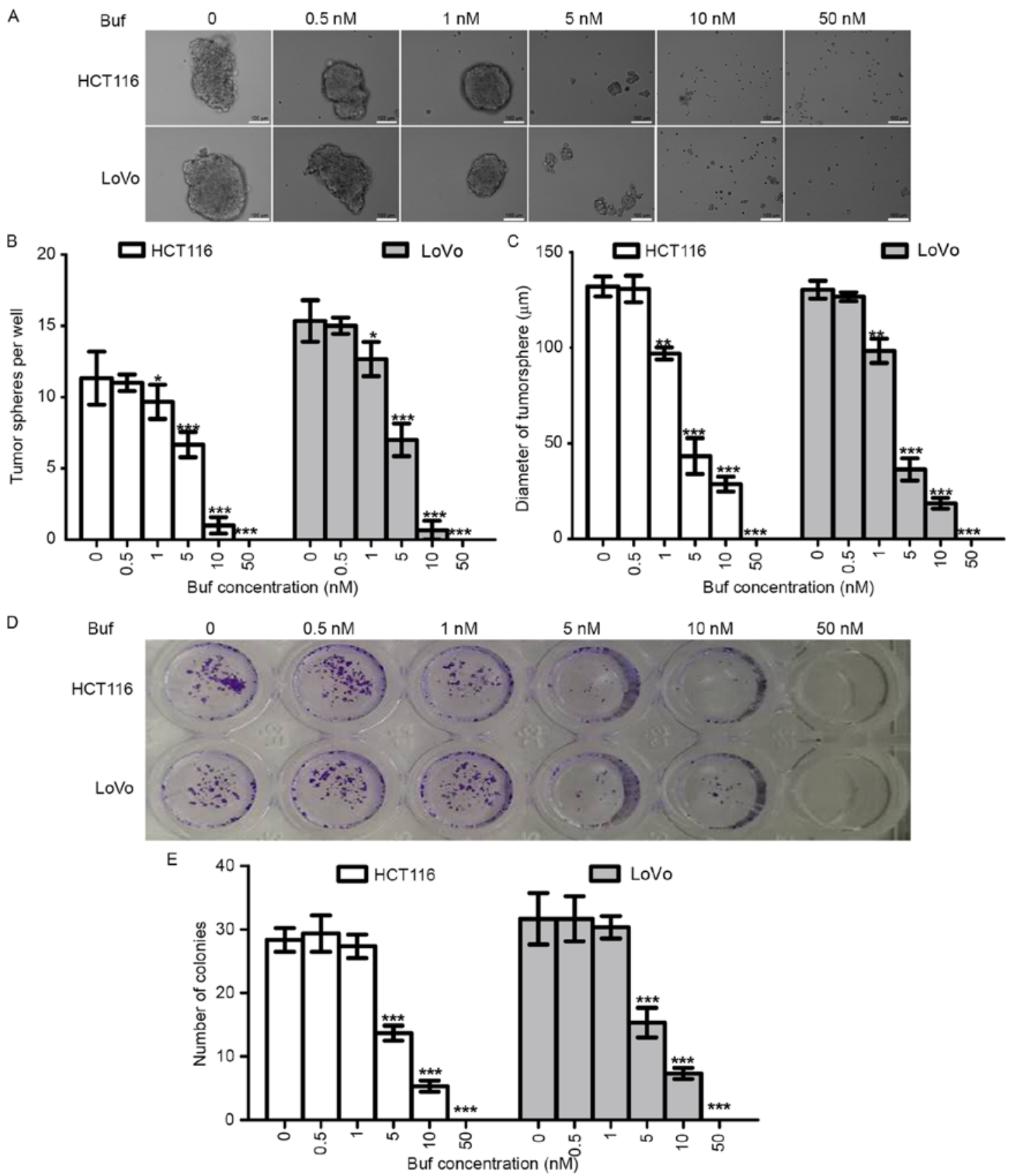

Figure 3. Effects of bufalin on tumorsphere formation in vitro. The tumorsphere formation assay was used to analyze the effects of bufalin $(0,0.5,1,5,10$ and $50 \mathrm{nM}$ ) on the stemness of HCT116 and LoVo cells. (A) Representative image of tumorspheres. (B) Histogram of the number of tumorspheres. (C) Histogram of tumorsphere diameter. (D) Representative image of colonies. (E) Histogram of the number of colonies. ${ }^{* * *} \mathrm{p}<0.001,{ }^{* *} \mathrm{p}<0.01,{ }^{*} \mathrm{p}<0.05$.

role of bufalin, we speculated that it would be effective against cisplatin with regard to the stemness of CRC cells. Primary tumorsphere cells treated by cisplatin $(5 \mu \mathrm{M})$, referred to as $\mathrm{PTSCs}^{\text {cis }}$, were used for the secondary tumorsphere formation assay (Fig. 4A-C). When compared with the control, we found that cisplatin promoted the formation of secondary tumorspheres, while bufalin alone decreased the formation of secondary tumorspheres. On the other hand, the combination of cisplatin and bufalin could inhibit the numbers and diameters of secondary tumorspheres relative to the control and cisplatin groups. These results suggested that bufalin works against cisplatin with regard to the stemness of CRC cells.

After the secondary tumorsphere formation assay, the ratios of SP cells were tested using flow cytometry through
Hoechst 33342 staining. As shown in Fig. 4D and E, the $\mathrm{SP} /$ total ratio increased in secondary tumorspheres treated with cisplatin relative to that of the control and decreased with the combination treatment or with bufalin alone. Moreover, Hoechst 33342-stained cells were photographed using a fluorescence microscope (Fig. 4F and G). The results of photography and flow cytometry corresponded with each other. These findings further confirmed the reversing effects of bufalin on an increase in stemness induced by cisplatin in CRC cells.

Bufalin antagonizes the effects of cisplatin with regard to the expression of stemness markers. Drug-treated cancer cells in the tumorsphere formation assay showed higher expression 

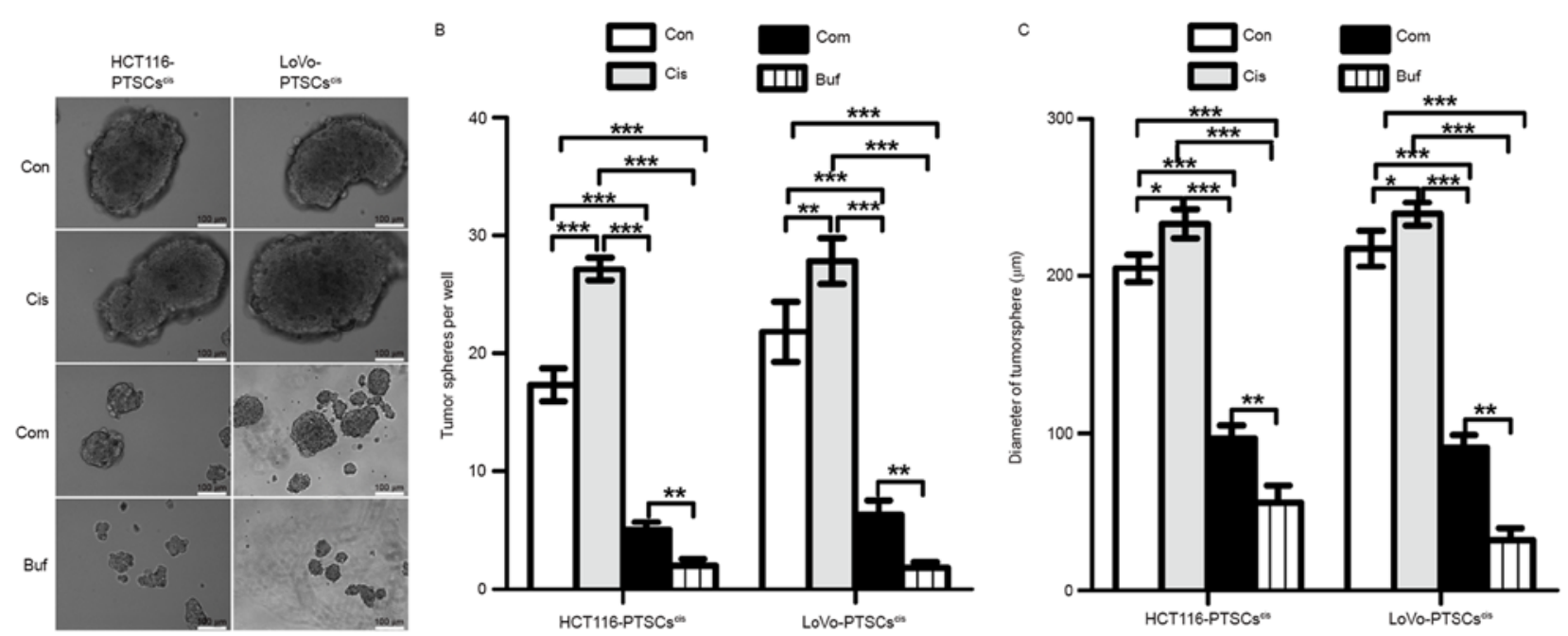

D

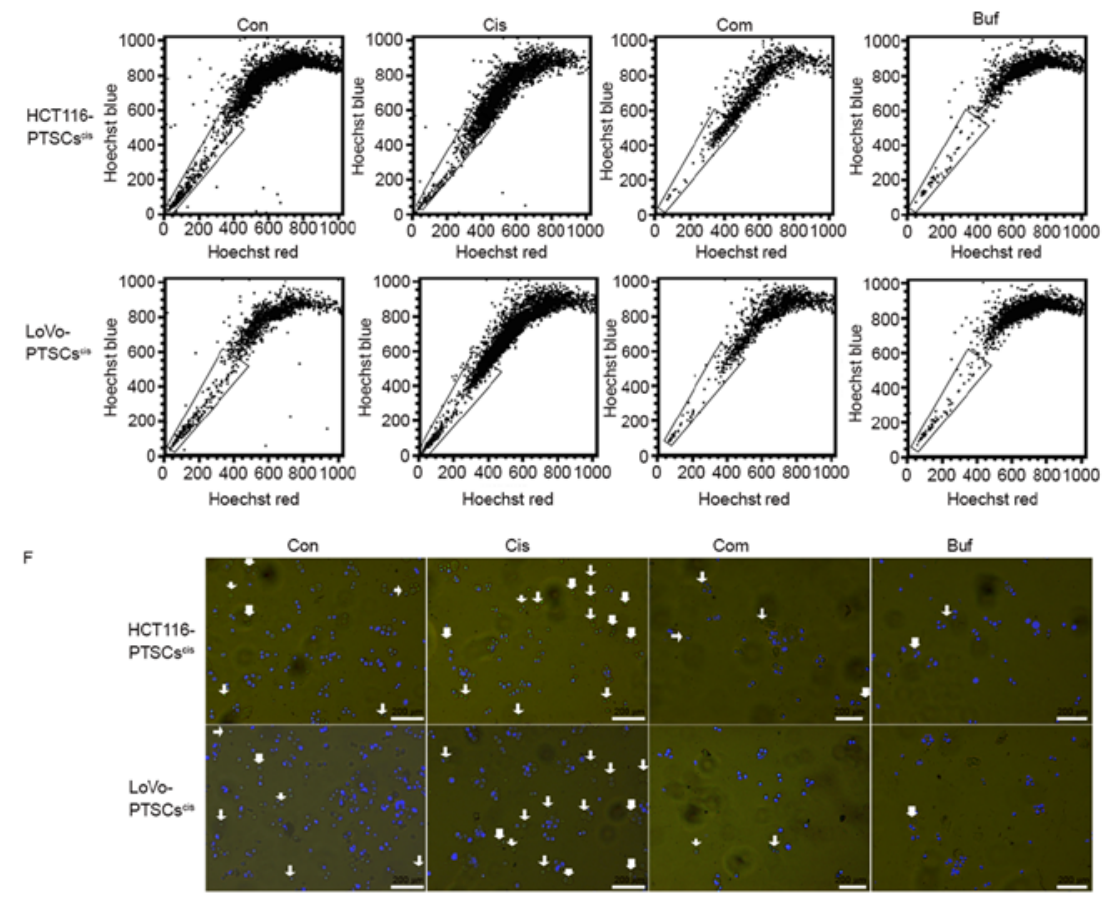

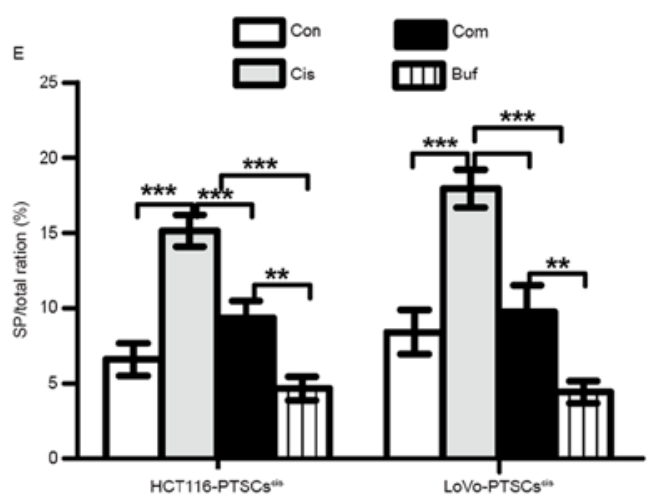

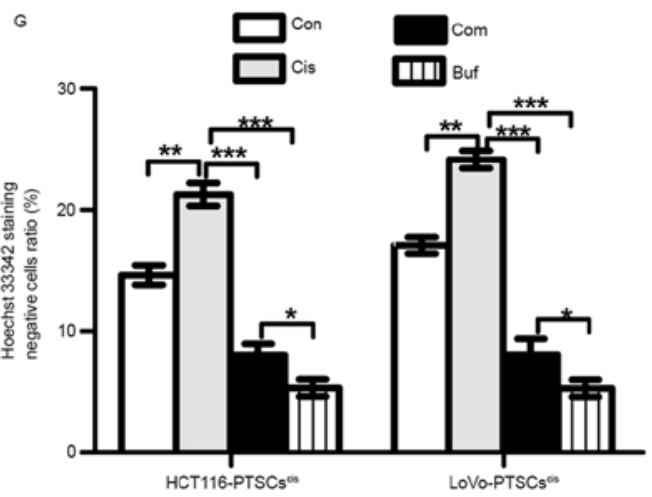

Figure 4. Bufalin is effective against cisplatin with regard to the stemness of colorectal cancer cells in vitro. The cells derived from PTSs treated with cisplatin $(5 \mu \mathrm{M})$, referred to as PTSCs ${ }^{\mathrm{cis}}$, are used for the secondary tumorsphere formation assay. These cells were treated with control (Con), $5 \mu \mathrm{M}$ cisplatin, $5 \mathrm{nM}$ bufalin, and combination (Com) separately. (A) Representative image of secondary tumorspheres. (B) Histogram of the number of secondary tumorspheres. (C) Histogram of secondary tumorsphere diameters. (D) SP ratio assay of STSCs using flow cytometry. (E) Histogram of the SP ratio. (F) Representative image of Hoechst 33342 staining of STSCs. (G) Histogram of the ratio of cells with negative staining for Hoechst $33342 .{ }^{* * * *} \mathrm{p}<0.001,{ }^{* *} \mathrm{p}<0.01,{ }^{*} \mathrm{p}<0.05$.

of stemness markers such as CD133, CD44, NANOG, OCT4, SOX2, and ABCG2 (36-39). Therefore, we tested the expression of these stemness markers in secondary tumorsphere cells using immunofluorescence, flow cytometry, and western blotting.

Initially, secondary tumorspheres were dissociated into single cells and were seeded in a 24 -well plate with slides. When most cells adhered to the slides, immunofluorescence assay was used to detect the expression and locations of the stemness markers in the cells. As shown in Fig. 5A, the expression of CD133, CD44, NANOG, OCT4, SOX2, and ABCG2 increased in the secondary tumorsphere cells treated with cisplatin alone. However, bufalin and combination treatment inhibited their expression.

At the same time, the two colorectal CSC markers CD133 and CD44 of secondary tumorsphere cells were assessed using flow cytometry (Fig. 5B). Consistent with the immunofluorescence results, bufalin antagonized the effects of cisplatin with regard to the expression of CD133 and CD44.

Finally, secondary tumorspheres underwent protein extraction to test the expression of stemness markers using western blotting. As shown in Fig. 5C, secondary tumorsphere cells treated with cisplatin (both HCT116 and LoVo cell lines) displayed higher expression of CD133, CD44, NANOG, 
A

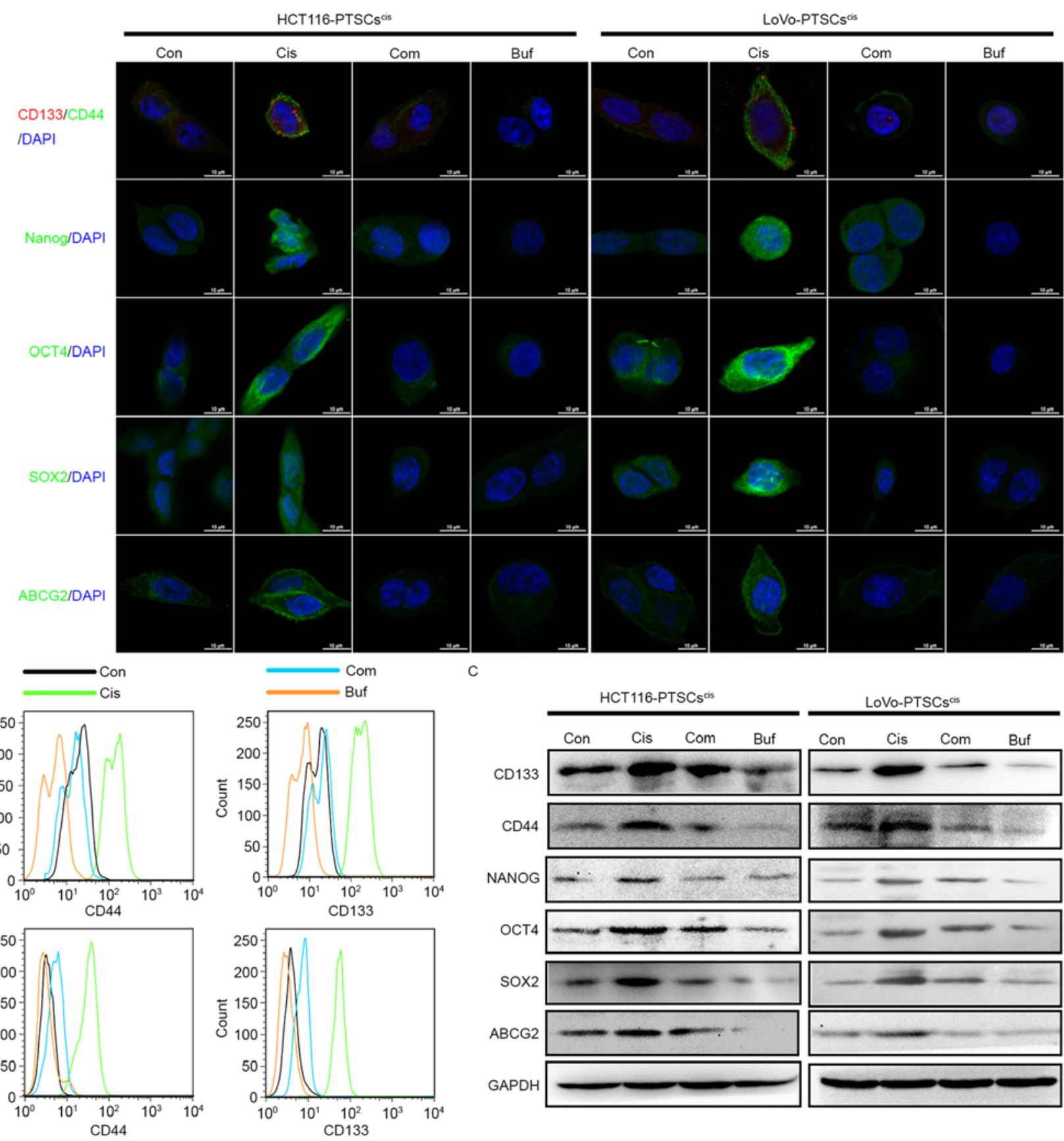

Figure 5. Bufalin antagonizes cisplatin with regard to the expression of stemness markers in colorectal cancer cells in vitro. (A) All secondary tumorspheres are dissociated into single cells and are seeded on cover slips in a 48-well plate. The protein expression of CD133, CD44, OCT4, SOX2, NANOG, and ABCG2 are evaluated by immunofluorescence. After 14 days, images of tumorspheres were obtained using microscopy. (B) The protein expression of CD133 and CD44 was evaluated using a flow cytometry histogram plot. (C) The protein expression of CD133, CD44, OCT4, SOX2, NANOG, and ABCG2 was evaluated and normalized with GAPDH using western blotting.

OCT4, SOX2, and ABCG2 proteins compared to the control. Bufalin decreased their protein expression alone. In addition, high expression of these proteins induced by cisplatin could be reversed by bufalin. These data further supported the effect of bufalin against cisplatin-induced stemness.

Bufalin reverses acquired drug resistance in colorectal cancer cells induced by cisplatin in vitro. Studies have shown that acquired drug resistance is associated with increased expression of stemness markers induced by chemotherapeutic drugs $(38,40,41)$. The results of this study also suggested that cisplatin increases the expression of stemness markers, while the effects of bufalin were the opposite. Therefore, we speculated that STSCs ${ }^{\text {cis }}$ had drug-resistant properties, while bufalin could inhibit this kind of acquired drug-resistance. To verify these speculations, we compared the sensitivity of STSCs ${ }^{\text {cis }}$ and parent cells to cisplatin. At the same time, we tested the synergistic effects of bufalin on the sensitivity of STSCs ${ }^{\text {cis }}$ to cisplatin. The STSCs ${ }^{\text {cis }}$ and their parent cells were seeded in $10 \%$ FBS RPMI-1640 medium at a density of $1 \times 10^{4}$ cells/well, in a 96-well plate. Then, $5 \mathrm{nM}$ bufalin and different concentrations of cisplatin were added for $48 \mathrm{~h}$. The results of the cell 

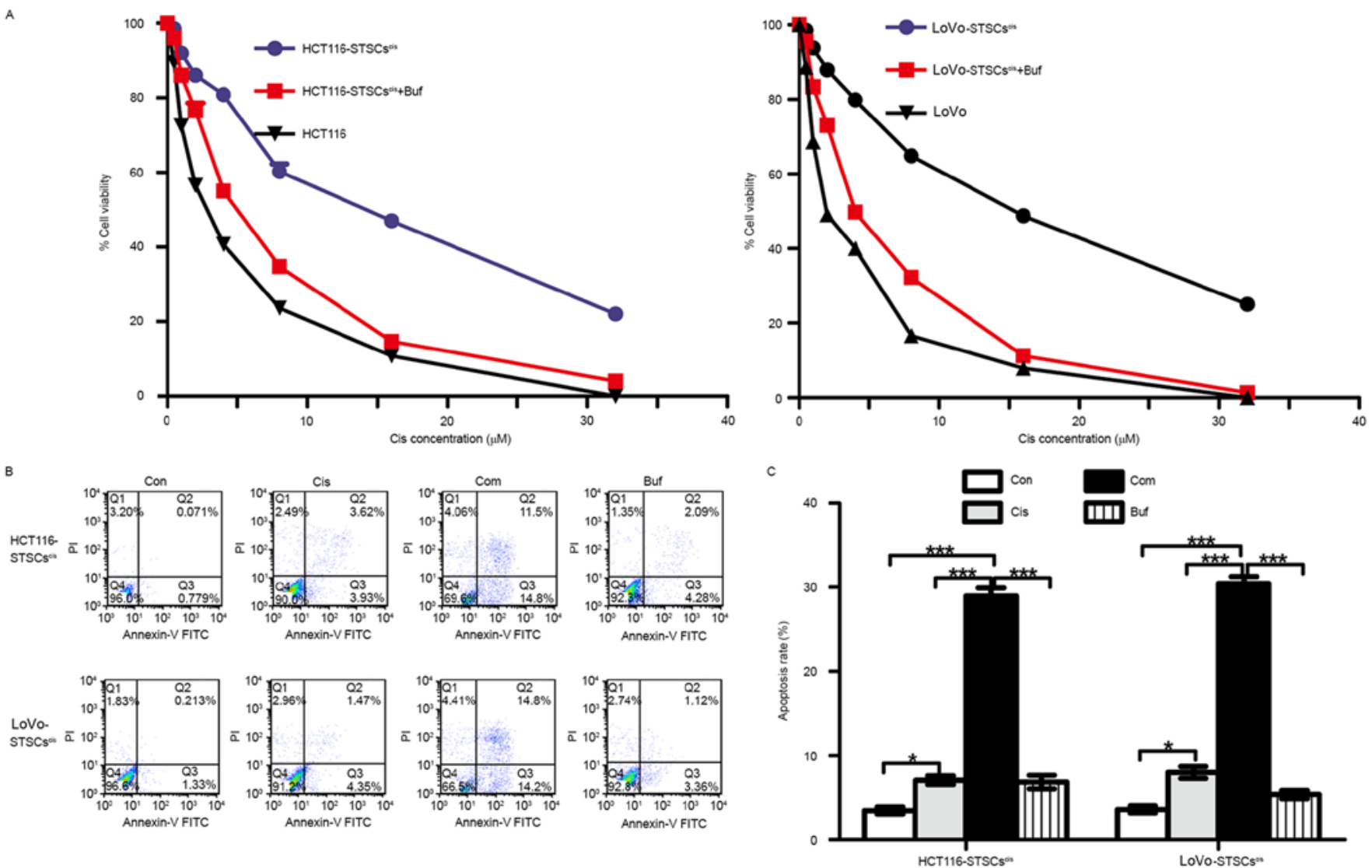

Figure 6. Bufalin improves the sensitivity of cisplatin in secondary tumorsphere cells treated with cisplatin (STSCs ${ }^{\mathrm{cis}}$ ). (A) $\mathrm{STSCs}^{\mathrm{cis}}$ and parent cells are seeded in a 96-well plate. The cell viability assay is used to analyze the drug-resistance of STSCs ${ }^{\text {cis }}$ and anti-drug resistance of bufalin. (B) STSCs ${ }^{\text {cis }}$ are treated with $5 \mu \mathrm{M}$ cisplatin, $5 \mathrm{nM}$ bufalin, and their combination for $48 \mathrm{~h}$, and the apoptosis rates are calculated. ${ }^{* * *} \mathrm{p}<0.001,{ }^{*} \mathrm{p}<0.05$.

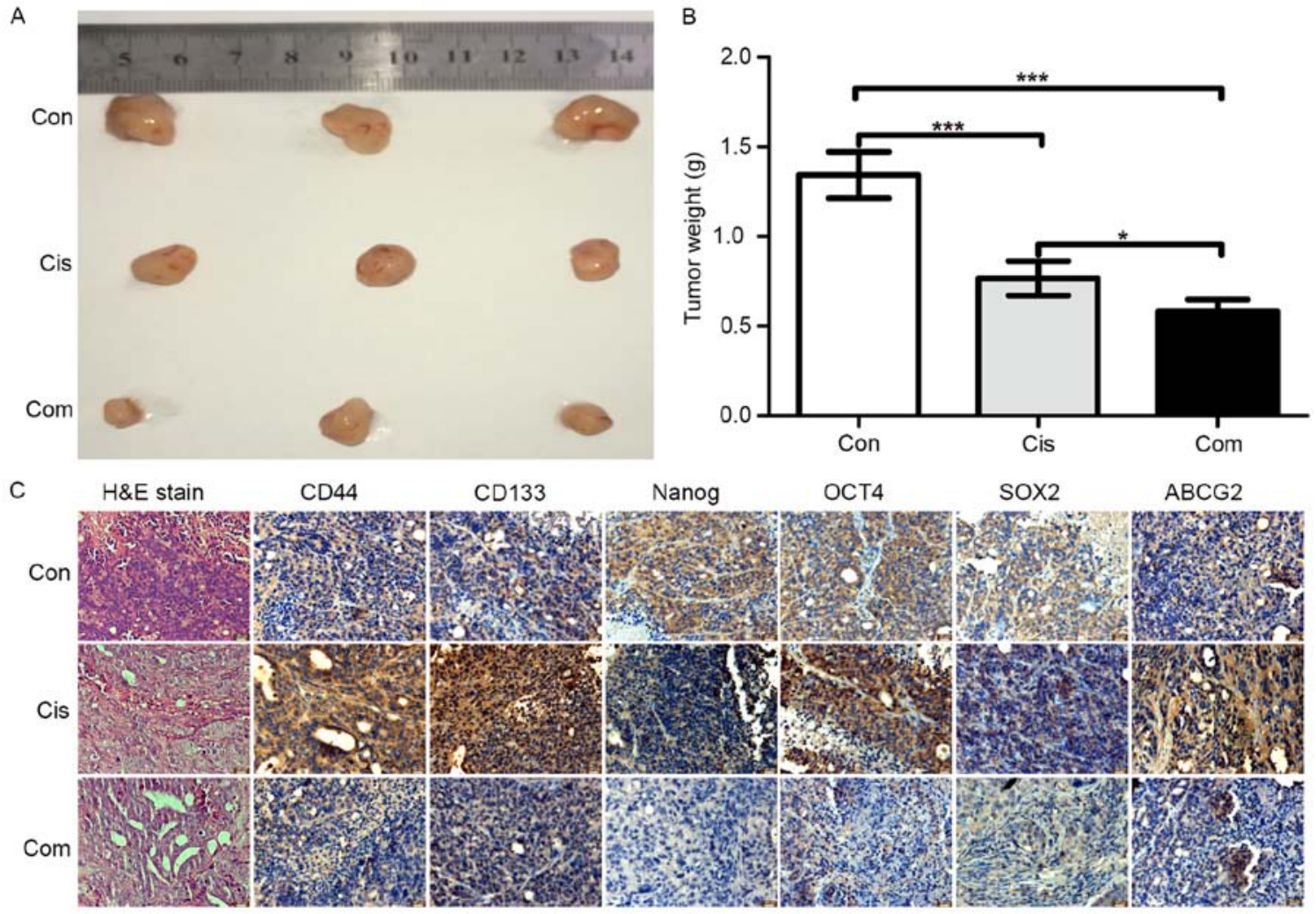

Figure 7. Bufalin synergistically inhibits the proliferation of colorectal cancer cells with cisplatin and decreases stemness in vivo. A subcutaneous xenograft model of HCT116 cells was treated with cisplatin alone or cisplatin + bufalin for 3 weeks. Tumors were photographed (A) and weighed (B). (C) Hematoxylin and eosin staining and immunohistochemistry were performed to evaluate the expression of stemness markers. 
viability assay using CCK8 are shown in Fig. 6A. The $\mathrm{IC}_{50}$ (concentration that produces $50 \%$ inhibition) of cisplatin in HCT116-STSCs ${ }^{\text {cis }}$ was $18.06 \pm 1.43 \mu \mathrm{M}$, which was higher than that of HCT116 cells $\left(\mathrm{IC}_{50}=2.13 \pm 0.12 \mu \mathrm{M}\right)$. Bufalin decreased the $\mathrm{IC}_{50}$ of HCT116-STSCs ${ }^{\text {cis }}$ to $5.61 \pm 0.42 \mu \mathrm{M}$. Similarly, the $\mathrm{IC}_{50}$ values of LoVo cells, LoVo-STSCs ${ }^{\text {cis }}$, and LoVoSTSCs $^{\text {cis }}$ treated with bufalin were $20.81 \pm 1.15,2.13 \pm 0.19$ and $4.9 \pm 0.23 \mu \mathrm{M}$, respectively.

Apoptosis assay involving flow cytometry was used to verify the results of the cell viability assay (Fig. 6B). STSCs ${ }^{\text {cis }}$ were treated with $5 \mathrm{mM}$ cisplatin, $5 \mathrm{nM}$ bufalin, and their combination for $48 \mathrm{~h}$, and the apoptosis rate was calculated. We found that the apoptosis rate with the combination was much higher than the rates with cisplatin and bufalin alone. The results further suggested that bufalin reverses the acquired drug-resistance induced by cisplatin in CRC cells.

Effect of bufalin on stemness marker expression induced by cisplatin in vivo. In vitro studies have shown that bufalin could inhibit stemness and increase the sensitivity of cisplatin in CRC cells. To investigate the anti-stemness effect of bufalin in vivo, a subcutaneous xenograft model of HCT116 cells in nude mice was used. HCT116 cells were subcutaneously injected into nude mice for 2 weeks, and then, the mice were treated with cisplatin alone or cisplatin + bufalin for 3 weeks. After sacrifice, the tumor tissues were weighed and paraffin-embedded tissue blocks were created for immunohistochemistry and H\&E staining. As shown in Fig. 7A and B, inhibition of tumor growth was greater with the combination of cisplatin and bufalin than with cisplatin alone. The tumor tissue weights also showed the synergistic effects of bufalin on cisplatin. According to the results of H\&E staining (Fig. 7C), tumors treated with the combination of cisplatin and bufalin showed more cell vacuolization and nuclear shrinkage than with cisplatin alone.

The expression of CD133, CD44, NANOG, OCT4, SOX2, and ABCG2 was assessed using immunohistochemistry to evaluate the effect of bufalin on stemness in vivo. Similar to the in vitro results, immunohistochemistry showed that cisplatin alone increased the protein expression of stemness markers (Fig. 7C), while the combination of cisplatin and bufalin decreased the protein expression of stemness markers. These results suggested that bufalin increased the sensitive of cisplatin in CRC cells through a reduction in stemness.

\section{Discussion}

Chemotherapy is a necessary treatment method after surgery in many advanced cancers. However, drug resistance has become a major obstacle to the successful treatment of cancer patients. Recent studies on molecular and cellular mechanisms have suggested that high stemness induced by chemotherapeutic drugs was an important reason for acquired drug-resistance. Therefore, many researchers have attempted to identify adjuvant chemotherapeutic drugs or new combinations of chemotherapeutic drugs that target tumor cell stemness.

Many chemotherapeutic drugs have been found to increase the stemness of cancer cells (42-46). In these studies, cisplatin, a type of platinum-based drug, was often used to investigate the relationship between acquired drug resistance and stemness
(42,47-52). The tumorsphere formation assay is an important method for verifying the stemness of cancer cells (34). In our study, we assessed the effects of cisplatin on tumorsphere formation in two CRC cell lines (HCT116 and LoVo). We found that cisplatin promoted tumorsphere formation. At the same time, the colony formation assay was used to analyze the effects of cisplatin on proliferation and apoptosis. However, we noted a reverse trend to that in the tumorsphere formation assay. The opposite results further supported the stemnessinducing effect of cisplatin in CRC cells. At present, traditional monolayer cultured cells show great differences from natural growth body cells in morphology, structure, function, and other aspects, which cannot really reflect the three-dimensional (3D) state of tumor growth in vivo. Therefore, a 3D cell culture system, such as the tumorsphere formation assay, is better suited for tumor invasion, metastasis, and drug-resistance research in vitro. In this study, low cisplatin concentrations (0.1-5 $\mu \mathrm{M})$ were found to increase the tumorsphere effects of CRC cells, consistent with other cancer cells $(40,42,48,50)$. Previous studies have shown the effects of bufalin on the inhibition of CSCs or stemness in pancreatic cancer cells and osteosarcoma CSCs (26-28). In our study, we also showed the effects of bufalin on the inhibition of stemness in CRC cells. Taking the same experiment, when treated by bufalin showed different results than cisplatin. Therefore, we speculated that bufalin could antagonize the increasing stemness induced by cisplatin in CRC cells. We used the secondary tumorsphere formation assay to test the inhibiting stemness effects of bufalin in CTSCs. We found that the combination of bufalin and cisplatin could inhibit tumorsphere formation, although the effect of bufalin alone was better.

The stemness of cancer cells can be represented with the CSC ratio, which could be evaluated with the SP ratio. Using the flow cytometry assay and imaging with microscopy, we assessed the Hoechst-negative SP ratio in the secondary tumorsphere assay. We found that cisplatin could increase the SP ratio, while bufalin inhibited the SP ratio. The SP cells can efflux out fluorescent dyes, such as the DNA-binding dye Hoechst 33342, which will cause the cells not to show staining under a fluorescence microscope or flow cytometry (53). Therefore, a high SP ratio induced by cisplatin represents a high CSC ratio or high stemness of CRC cells.

High stemness was often accompanied by drug resistance in cancer cells. We assessed the drug resistance of STSCs ${ }^{\text {cis }}$. We found that drug resistance was higher in $\mathrm{STSCs}^{\mathrm{cis}}$ than in their parent cells, which proved that cisplatin could induce acquired drug resistance. In view of the inhibiting stemness and acquired drug-resistance effects (23), we speculated that bufalin could inhibit acquired cisplatin resistance in CRC cells via the inhibition of stemness. We found that the combination of bufalin and cisplatin could inhibit proliferation and induce apoptosis in STSCs ${ }^{\text {cis }}$ in vitro. The combination of bufalin and cisplatin showed higher effects than cisplatin alone in vivo. These results verified our speculation that the reversion effects of bufalin on acquired cisplatin resistance relied on the inhibition of stemness in CRC.

Recent studies suggested that cisplatin induces high expression of stemness markers such as CD133 (49), CD44 (47), NANOG (50), SOX2 (50), OCT4 (50), and ABCG2 (49). Therefore, we assessed the expression of stemness markers 
in secondary tumorsphere cells. We found that cisplatin could promote high expression of these markers of CRC cells in vitro and in vivo, while bufalin could antagonize the effect of cisplatin on the expression of these markers. These results further supported our initial hypothesis that bufalin could reverse acquired cisplatin resistance via the inhibition of stemness in CRC cells.

In this study, we verified that the ability of bufalin to reverse acquired cisplatin resistance relied on the inhibition of stemness in CRC cells. These findings provide information for new chemotherapy strategies for the clinical treatment of CRC. In addition, these findings remind oncologists to include agents than can inhibit the stemness effect to prevent acquired drug resistance in tumor chemotherapy. The specific molecular mechanisms are not very clear and require further research.

In conclusion, bufalin can reverse acquired cisplatin resistance both in vitro and in vivo by inhibiting the stemness of $\mathrm{CRC}$ and decreasing the expression of stemness markers, such as CD133, CD44, OCT4, SOX2, and NANOG, and the drugresistant protein $\mathrm{ABCG} 2$. These findings suggest that bufalin plays an adjuvant role in CRC chemotherapy and may help reverse acquired drug resistance.

\section{Acknowledgements}

This study was supported by the National Natural Science Foundation of China (nos. 81473482 and 81503434).

\section{References}

1. Siegel RL, Miller KD and Jemal A: Cancer statistics, 2016. CA Cancer J Clin 66: 7-30, 2016

2. Edwards BK, Ward E, Kohler BA, Eheman C, Zauber AG, Anderson RN, Jemal A, Schymura MJ, Lansdorp-Vogelaar I, Seeff LC, et al: Annual report to the nation on the status of cancer, 1975-2006, featuring colorectal cancer trends and impact of interventions (risk factors, screening, and treatment) to reduce future rates. Cancer 116: 544-573, 2010.

3. Chen W, Zheng R, Baade PD, Zhang S, Zeng H, Bray F, Jemal A, Yu XQ and He J: Cancer statistics in China, 2015. CA Cancer J Clin 66: 115-132, 2016.

4. Center MM, Jemal A and Ward E: International trends in colorectal cancer incidence rates. Cancer Epidemiol Biomarkers Prev 18: 1688-1694, 2009.

5. Center MM, Jemal A, Smith RA and Ward E: Worldwide variations in colorectal cancer. CA Cancer J Clin 59: 366-378, 2009.

6. Jemal A, Bray F, Center MM, Ferlay J, Ward E and Forman D: Global cancer statistics. CA Cancer J Clin 61: 69-90, 2011.

7. Ramasamy TS, Ayob AZ, Myint HHL, Thiagarajah S and Amini F: Targeting colorectal cancer stem cells using curcumin and curcumin analogues: Insights into the mechanism of the therapeutic efficacy. Cancer Cell Int 15: 96, 2015.

8. Donnenberg VS and Donnenberg AD: Multiple drug resistance in cancer revisited: The cancer stem cell hypothesis. J Clin Pharmacol 45: 872-877, 2005.

9. Dean M: ABC transporters, drug resistance, and cancer stem cells. J Mammary Gland Biol Neoplasia 14: 3-9, 2009.

10. Clarke MF, Dick JE, Dirks PB, Eaves CJ, Jamieson CH, Jones DL, Visvader J, Weissman IL and Wahl GM: Cancer stem cells - perspectives on current status and future directions: AACR Workshop on cancer stem cells. Cancer Res 66: 9339-9344, 2006.

11. Visvader JE and Lindeman GJ: Cancer stem cells in solid tumours: Accumulating evidence and unresolved questions. Nat Rev Cancer 8: 755-768, 2008.

12. Dalerba P, Dylla SJ, Park I-K, Liu R, Wang X, Cho RW, Hoey T, Gurney A, Huang EH, Simeone DM, et al: Phenotypic characterization of human colorectal cancer stem cells. Proc Natl Acad Sci USA 104: 10158-10163, 2007.
13. Ricci-Vitiani L, Lombardi DG, Pilozzi E, Biffoni M, Todaro M, Peschle $C$ and De Maria R: Identification and expansion of human colon-cancer-initiating cells. Nature 445: 111-115, 2007.

14. Lugli A, Iezzi G, Hostettler I, Muraro MG, Mele V, Tornillo L, Carafa V, Spagnoli G, Terracciano L and Zlobec I: Prognostic impact of the expression of putative cancer stem cell markers CD133, CD166, CD44s, EpCAM, and ALDH1 in colorectal cancer. Br J Cancer 103: 382-390, 2010.

15. Meng H-M, Zheng P, Wang X-Y, Liu C, Sui H-M, Wu S-J, Zhou J, Ding Y-Q and Li J: Over-expression of Nanog predicts tumor progression and poor prognosis in colorectal cancer. Cancer Biol Ther 9: 295-302, 2010.

16. Saigusa S, Tanaka K, Toiyama Y, Yokoe T, Okugawa Y, Ioue Y, Miki C and Kusunoki M: Correlation of CD133, OCT4, and SOX2 in rectal cancer and their association with distant recurrence after chemoradiotherapy. Ann Surg Oncol 16: 3488-3498, 2009.

17. Jeter CR, Liu B, Liu $X$, Chen $X$, Liu C, Calhoun-Davis $T$, Repass J, Zaehres H, Shen JJ and Tang DG: NANOG promotes cancer stem cell characteristics and prostate cancer resistance to androgen deprivation. Oncogene 30: 3833-3845, 2011.

18. Kodach LL, Jacobs RJ, Voorneveld PW, Wildenberg ME, Verspaget HW, van Wezel T, Morreau H, Hommes DW, Peppelenbosch MP, van den Brink GR, et al: Statins augment the chemosensitivity of colorectal cancer cells inducing epigenetic reprogramming and reducing colorectal cancer cell 'stemness' via the bone morphogenetic protein pathway. Gut 60: 1544-1553, 2011.

19. Yin PH, Liu X, Qiu YY, Cai JF, Qin JM, Zhu HR and Li Q: Antitumor activity and apoptosis-regulation mechanisms of bufalin in various cancers: New hope for cancer patients. Asian Pac J Cancer Prev 13: 5339-5343, 2012.

20. Wang J, Chen C, Wang S, Zhang Y, Yin P, Gao Z, Xu J, Feng D, Zuo Q, Zhao R, et al: Bufalin inhibits HCT116 colon cancer cells and its orthotopic xenograft tumor in mice model through genes related to apoptotic and PTEN/AKT pathways. Gastroenterol Res Pract 2015: 457193, 2015.

21. Qiu YY, Hu Q, Tang QF, Feng W, Hu SJ, Liang B, Peng W and Yin PH: MicroRNA-497 and bufalin act synergistically to inhibit colorectal cancer metastasis. Tumour Biol 35: 2599-2606, 2014.

22. Liu T, Jia T, Yuan X, Liu C, Sun J, Ni Z, Xu J, Wang X and Yuan Y: Development of octreotide-conjugated polymeric prodrug of bufalin for targeted delivery to somatostatin receptor 2 overexpressing breast cancer in vitro and in vivo. Int J Nanomed 11: 2235-2250, 2016.

23. Zhao H, Zhao D, Jin H, Li H, Yang X, Zhuang L and Liu T: Bufalin reverses intrinsic and acquired drug resistance to cisplatin through the AKT signaling pathway in gastric cancer cells. Mol Med Rep 14: 1817-1822, 2016.

24. Wu SH, Bau DT, Hsiao YT, Lu KW, Hsia TC, Lien JC, Ko YC Hsu WH, Yang ST, Huang YP, et al: Bufalin induces apoptosis in vitro and has Antitumor activity against human lung cancer xenografts in vivo. Environ Toxicol 32: 1305-1317, 2017.

25. Xie CM, Chan WY, Yu S, Zhao J and Cheng CH: Bufalin induces autophagy-mediated cell death in human colon cancer cells through reactive oxygen species generation and JNK activation. Free Radic Biol Med 51: 1365-1375, 2011.

26. Chang Y, Zhao Y, Gu W, Cao Y, Wang S, Pang J and Shi Y: Bufalin inhibits the differentiation and proliferation of cancer stem cells derived from primary osteosarcoma cells through mir-148a. Cell Physiol Biochem 36: 1186-1196, 2015.

27. Chang Y, Zhao Y, Zhan H, Wei X, Liu T and Zheng B: Bufalin inhibits the differentiation and proliferation of human osteosarcoma cell line hMG63-derived cancer stem cells. Tumour Biol 35: 1075-1082, 2014.

28. Wang H, Ning Z, Li Y, Zhu X and Meng Z: Bufalin suppresses cancer stem-like cells in gemcitabine-resistant pancreatic cancer cells via Hedgehog signaling. Mol Med Rep 14: 1907-1914, 2016.

29. Gai JQ, Sheng X, Qin JM, Sun K, Zhao W and Ni L: The effect and mechanism of bufalin on regulating hepatocellular carcinoma cell invasion and metastasis via Wnt/ $\beta$-catenin signaling pathway. Int J Oncol 48: 338-348, 2016.

30. Zhu Z, Sun H, Ma G, Wang Z, Li E, Liu Y and Liu Y: Bufalin induces lung cancer cell apoptosis via the inhibition of PI3K/Akt pathway. Int J Mol Sci 13: 2025-2035, 2012.

31. Zhu Z, Li E, Liu Y, Gao Y, Sun H, Ma G, Wang Z, Liu X, Wang Q, Qu X, et al: Inhibition of Jak-STAT3 pathway enhances bufalin-induced apoptosis in colon cancer SW620 cells. World J Surg Oncol 10: 228, 2012. 
32. Bertrand FE, Angus CW, Partis WJ and Sigounas G: Developmental pathways in colon cancer: Crosstalk between WNT, BMP, Hedgehog and Notch. Cell Cycle 11: 4344-4351, 2012.

33. Cao L, Zhou Y, Zhai B, Liao J, Xu W, Zhang R, Li J, Zhang Y, Chen L, Qian H, et al: Sphere-forming cell subpopulations with cancer stem cell properties in human hepatoma cell lines. BMC Gastroenterol 11: 71, 2011.

34. Chen S-F, Chang Y-C, Nieh S, Liu C-L, Yang C-Y and Lin Y-S: Nonadhesive culture system as a model of rapid sphere formation with cancer stem cell properties. PLoS One 7: e31864, 2012.

35. Jung MJ, Rho JK, Kim YM, Jung JE, Jin YB, Ko YG, Lee JS, Lee SJ, Lee JC and Park MJ: Upregulation of CXCR4 is functionally crucial for maintenance of stemness in drug-resistant non-small cell lung cancer cells. Oncogene 32: 209-221, 2013.

36. Levina V, Marrangoni AM, DeMarco R, Gorelik E and Lokshin AE: Drug-selected human lung cancer stem cells: Cytokine network, tumorigenic and metastatic properties. PLoS One 3: e3077, 2008.

37. Hamilton $\mathrm{G}$ and Olszewski U: Chemotherapy-induced enrichment of cancer stem cells in lung cancer. J Bioanal Biomed: S9, 2013. doi:10.4172/1948-593X.S9-003.

38. Sun FF, Hu YH, Xiong LP, Tu XY, Zhao JH, Chen SS, Song J and Ye XQ: Enhanced expression of stem cell markers and drug resistance in sphere-forming non-small cell lung cancer cells. In J Clin Exp Pathol 8: 6287-6300, 2015.

39. Liu J, Wang L, Ma L, Xu J, Liu C, Zhang J, Liu J and Chen R: Significantly increased expression of OCT4 and ABCG2 in spheroid body-forming cells of the human gastric cancer MKN-45 cell line. Oncol Lett 6: 891-896, 2013.

40. Abubaker K, Latifi A, Luwor R, Nazaretian S, Zhu H, Quinn MA, Thompson EW, Findlay JK and Ahmed N: Short-term single treatment of chemotherapy results in the enrichment of ovarian cancer stem cell-like cells leading to an increased tumor burden. Mol Cancer 12: 24, 2013

41. Vidal SJ, Rodriguez-Bravo V, Galsky M, Cordon-Cardo C and Domingo-Domenech J: Targeting cancer stem cells to suppress acquired chemotherapy resistance. Oncogene 33: 4451-4463, 2014.

42. Wiechert A, Saygin C, Thiagarajan PS, Rao VS, Hale JS, Gupta N, Hitomi M, Nagaraj AB, DiFeo A, Lathia JD, et al: Cisplatin induces stemness in ovarian cancer. Oncotarget 7: 30511-30522, 2016.

43. Bu Y, Jia Q-A, Ren Z-G, Zhang J-B, Jiang X-M, Liang L, Xue T-C, Zhang Q-B, Wang Y-H, Zhang L, et al: Maintenance of stemness in oxaliplatin-resistant hepatocellular carcinoma is associated with increased autocrine of IGF1. PLoS One 9: e89686, 2014.
44. Ress AL, Stiegelbauer V, Schwarzenbacher D, Deutsch A, Perakis S, Ling H, Ivan C, Calin GA, Rinner B, Gerger A, et al: Spinophilin expression determines cellular growth, cancer stemness and 5-flourouracil resistance in colorectal cancer. Oncotarget 5: 8492-8502, 2014.

45. Zhang Z, Duan Q, Zhao H, Liu T, Wu H, Shen Q, Wang C and Yin T: Gemcitabine treatment promotes pancreatic cancer stemness through the Nox/ROS/NF- $\mathrm{B} / \mathrm{STAT} 3$ signaling cascade. Cancer Lett 382: 53-63, 2016.

46. Ayadi M, Bouygues A, Ouaret D, Ferrand N, Chouaib S, Thiery J-P, Muchardt C, Sabbah M and Larsen AK: Chronic chemotherapeutic stress promotes evolution of stemness and WNT/beta-catenin signaling in colorectal cancer cells: Implications for clinical use of WNT-signaling inhibitors. Oncotarget 6: 18518-18533, 2015.

47. Nör C, Zhang Z, Warner KA, Bernardi L, Visioli F, Helman JI, Roesler R and Nör JE: Cisplatin induces Bmi-1 and enhances the stem cell fraction in head and neck cancer. Neoplasia 16: 137-146, 2014.

48. Chowanadisai W, Messerli SM, Miller DH, Medina JE, Hamilton JW, Messerli MA and Brodsky AS: Cisplatin resistant spheroids model clinically relevant survival mechanisms in ovarian tumors. PLoS One 11: e0151089, 2016.

49. Liu YP, Yang CJ, Huang MS, Yeh CT, Wu AT, Lee YC, Lai TC, Lee $\mathrm{CH}$, Hsiao YW, Lu J, et al: Cisplatin selects for multidrugresistant $\mathrm{CD} 133^{+}$cells in lung adenocarcinoma by activating Notch signaling. Cancer Res 73: 406-416, 2013.

50. Zhang F, Duan S, Tsai Y, Keng PC, Chen Y, Lee SO and Chen Y: Cisplatin treatment increases stemness through upregulation of hypoxia-inducible factors by interleukin- 6 in non-small cell lung cancer. Cancer Sci 107: 746-754, 2016.

51. Yang J, Guo W, Wang L, Yu L, Mei H, Fang S, Ji P, Liu Y, Liu G and Song Q: Cisplatin-resistant osteosarcoma cells possess cancer stem cell properties in a mouse model. Oncol Lett 12: 2599-2605, 2016

52. Tsai LL, Yu CC, Chang YC, Yu CH and Chou MY: Markedly increased Oct 4 and Nanog expression correlates with cisplatin resistance in oral squamous cell carcinoma. J Oral Pathol Med 40: 621-628, 2011.

53. She JJ, Zhang PG, Wang X, Che XM and Wang ZM: Side population cells isolated from KATO III human gastric cancer cell line have cancer stem cell-like characteristics. World J Gastroenterol 18: 4610-4617, 2012 